\title{
Mesoporous Carbon Structure Directed by Mesostructured Cellular Foam Silica
}

\author{
Yukito ODA, ${ }^{a}$ Seitaro NAMBA, ${ }^{b}$ Hideaki YOSHITAKE, ${ }^{c}$ and Takashi TATSUMI ${ }^{a}$
}

\author{
${ }^{a}$ Graduate School of Engineering, Yokohama National University (Tokiwadai, Hodogaya-ku, Yokohama 240-8501, \\ Japan) \\ ${ }^{b}$ Department of Materials, Teikyo University of Science \& Technology (Uenohara-machi, Kitatsuru-gun, Yamanashi \\ 409-0193, Japan) \\ 'Graduate School of Environment and Information Sciences, Yokohama National University (Tokiwadai, Hodogaya-ku, \\ Yokohama 240-8501, Japan)
}

Received June 12, 2002 ; Accepted September 25, 2002

\begin{abstract}
We synthesised a new mesoporous carbon consisting of aggregates of uniformly sized spherical particles are aggregated. The structure is directed by the carbonisation of sucrose in the pores of mesocellular foam silica. The BET specific surface area and the transmission electron micrograph demonstrate that the carbon particles are not solid spheres but hollow ones whose diameter and the thickness of the wall are 30 and $3 \mathrm{~nm}$, respectively.
\end{abstract}

Key Words : Mesoporous Carbon, Mesostructured Cellular Foam, Template

\section{Introduction}

For many years the development of micro fuel cells has been an urgent issue in the electronic power supply for mobile devices. The charge transfer reaction between the molecules and the electrode occurs at the interface in a membrane-electrode assembly (MEA), which is composed of metal particles, a proton conductive polymer and a carbon substrate. Since these elements have been simply assembled so that the cell works efficiently, the ill-defined structure prevents the chemists from investigating the electrochemical reactions and miniaturizing fuel cells. Manipulative preparation of the carbon substrate on a mesoscopic scale is indispensable for changing this situation. This is because the reaction processes during the conversion of gas molecules into ions are accompanied by a mesoscopic transfer of the molecule, adsorbates and chains of the polymer electrolyte.

The recent successes in syntheses of mesoporous silica with micelle templates have stimulated the chemistry of mesostructured materials. ${ }^{1-4)}$ Through the dehydration and subsequent pyrolysis of $\mathrm{OH}$-containing organic compounds, in the pores of mesoporous silica the pores are filled with carbon. The dissolution of silica with $\mathrm{HF}$ or $\mathrm{NaOH}$ removes "template mesoporous silica" from the composite, yielding mesoporous carbon obtained. This synthetic strategy of mesostructure by ordered carbon has been developed by Korean research groups. ${ }^{5-13)}$ The advantage of this method is that various well-defined structures of mesoporous silica can be used as the moulds. Mesoporous silica such as MCM-48 ${ }^{6}$ and SBA$15^{11)}$ are frequently used as templates. The structure of the carbons directed by such silicas are well ordered in a hexagonal array, while the spherical carbons aggregated in a mesoscale order have not yet been synthesized. We used mesostructured cellular foam silica (MCF-Si) synthesized recently ${ }^{14^{-16}}$ as a template and succeeded in the transfer of the mesostructure from silica to carbon. This communication reports the synthesis and characterisation of mesostructured cellular foam carbon (MCF-C).

\section{Experimental}

All the chemicals were used as received without further purification. The MCF-Si was prepared in hydrochloric acid using Pluronic P123 triblock copolymer surfactant (distributed by BASF) with 1, 3, 5-trimethylbenzene (TMB) as the swelling agent. Pluronic P123 was dissolved in $1.6 \mathrm{M} \mathrm{HCl}$ at room temperature while being stirred in a beaker. After TMB and $\mathrm{NH}_{4} \mathrm{~F}$ were added and the solution was heated to $313 \mathrm{~K}$, was added tetraethyl orthosilicate (TEOS). The reaction mixture was transferred to an autoclave and aged at $373 \mathrm{~K}$ for $24 \mathrm{~h}$ under static conditions. The molar composition was 0.4 Pluronic P123 : 21TEOS : 120 1.6 M HCl : 17 TMB : 0.9 $\mathrm{NH}_{4} \mathrm{~F}$. The mixture was then allowed to cool to room temperature and the precipitate was isolated by filtration, washed, dried in air for 2 days and calcined at $773 \mathrm{~K}$ for $8 \mathrm{~h}$ in air.

To a solution of $1.88 \mathrm{~g}$ of sucrose and $0.21 \mathrm{~g}$ of sulphuric acid in $5 \mathrm{~g}$ water was added $1 \mathrm{~g}$ of MCF-Si. The mixture was placed in an oven at $373 \mathrm{~K}$ for $6 \mathrm{~h}$ and subsequently the temperature was raised to $433 \mathrm{~K}$ and kept for $6 \mathrm{~h}$. The resulting dark brown powder was treated again in a similar manner by impregnation with $1.13 \mathrm{~g}$ of sucrose, $0.12 \mathrm{~g}$ of sulphuric acid in $5 \mathrm{~g}$ of water followed by drying-heating in an oven. The carbonisation was completed by pyrolysis at $1173 \mathrm{~K}$ under an argon flow. The carbon-silica composite thus obtained was washed with $4.7 \mathrm{wt} \%$ hydrofluoric acid at room temperature to 
remove silica. The templated-free carbon was collected by filtration, washed with ethanol and dried at $393 \mathrm{~K}$.

Nitrogen adsorption-desorption isotherms were recorded on a BELSORP 28SA porosimeter (BEL Japan Inc.) after the sample was evacuated at $473 \mathrm{~K}$ for $2 \mathrm{~h}$. Transmission electron micrographic (TEM) images were obtained with a JEM-2000EX (JEOL) operated at 200 keV.

\section{Results and Discussion}

The nitrogen adsorption isotherms and $\mathrm{DH}$ pore size distributions for MCF-Si are shown in Fig. 1. The adsorption branch shows a steep increase at the relative pressure $P / P_{o}$ at 0.82 , suggesting the presence of relatively large mesopores. The hysteresis implies an inkbottle structure of the pores. The most probable pore sizes determined from adsorption and desorption branches are

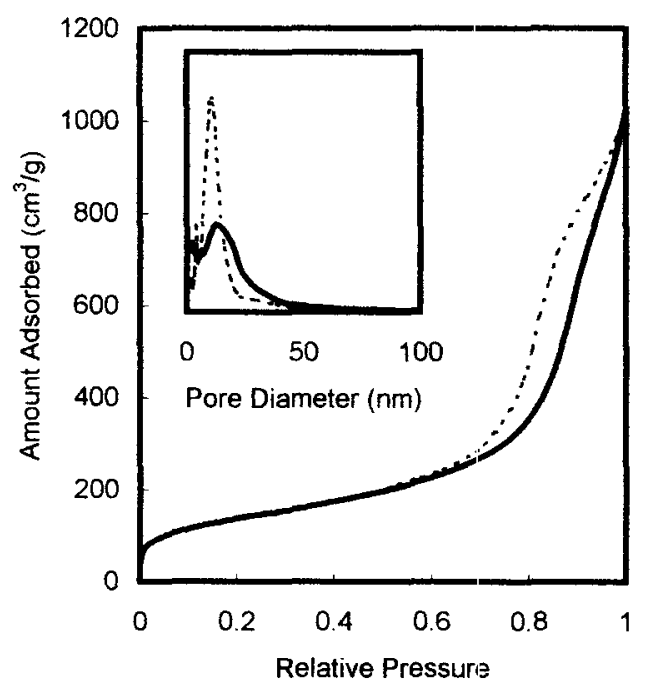

Fig. 1 Nitrogen adsorption-desorption isotherms and pore size distributions (inset) of MCF-Si. Solid and broken lines are adsorption and desorption branches, respectively. BET surface area and pore volume are $463 \mathrm{~m}^{2} \mathrm{~g}^{-1}$ and $1.47 \mathrm{~cm}^{3}$ $\mathrm{g}^{-1}$, respectively.

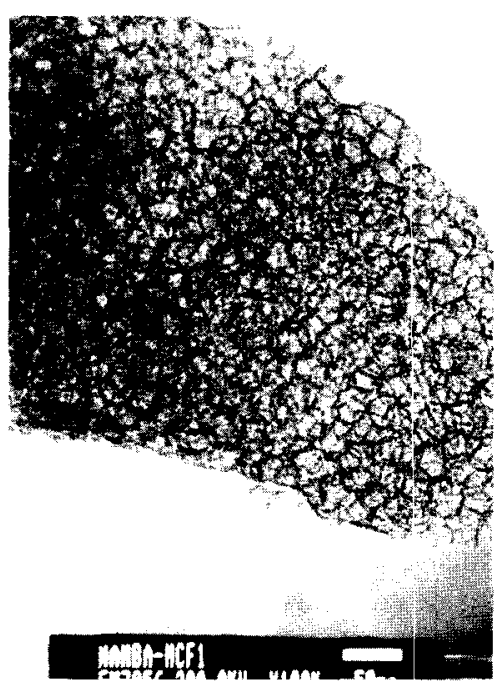

Fig. 2 Transmission electron micrograph of MCF-Si. The length of white bar is $50 \mathrm{~nm}$.
15.3 and $11.9 \mathrm{~nm}$, respectively, which could represent the diameters of the spherical pores and the mouth of the pores (the juncture of the pores), respectively. These dimensions agree well with those evaluated from the TEM photograph of MCF-Si shown in Fig. 2. The thickness of the pore wall is uniform at $3.3 \mathrm{~nm}$. The BET surface area and the total pore volume are $463 \mathrm{~m}^{2} \mathrm{~g}^{-1}$ and $1.47 \mathrm{~cm}^{3} \mathrm{~g}^{-1}$, respectively.

The nitrogen adsorption isotherms of mesoporous carbon templated by MCF-Si are shown in Fig. 3. The adsorption isotherm shows a gap at the relative pressure $P$ $/ P_{O}$ of 0.75 , suggesting that the mesopores of the carbon are smaller than those of the parent MCF-Si. The hysteresis is observed as in the case of MCF-Si and the DH pore size distributions are represented by the peak tops

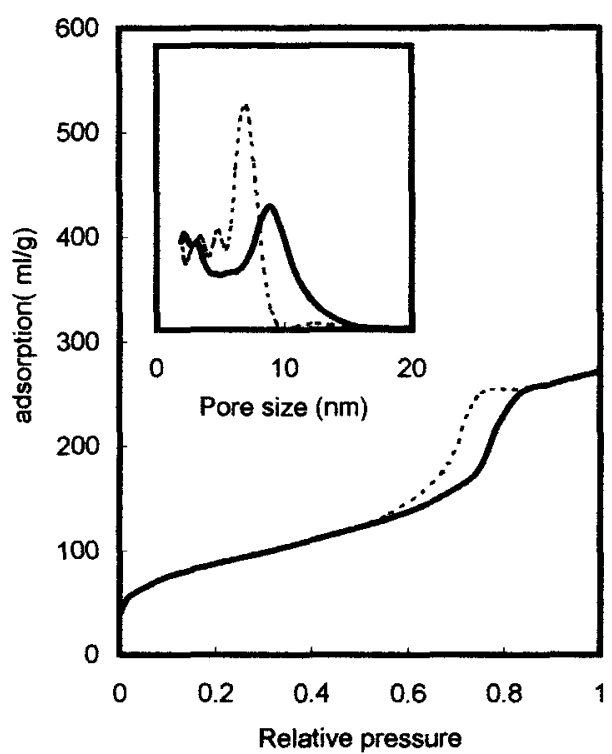

Fig. 3 Nitrogen adsorption-desorption isotherms and pore size distributions (inset) of MCF-C. Solid and broken lines are adsorption and desorption branches, respectively. BET surface area and pore volume are $291 \mathrm{~m}^{2} \mathrm{~g}^{-1}$ and $0.39 \mathrm{~cm}^{3}$ $\mathrm{g}^{-1}$, respectively.

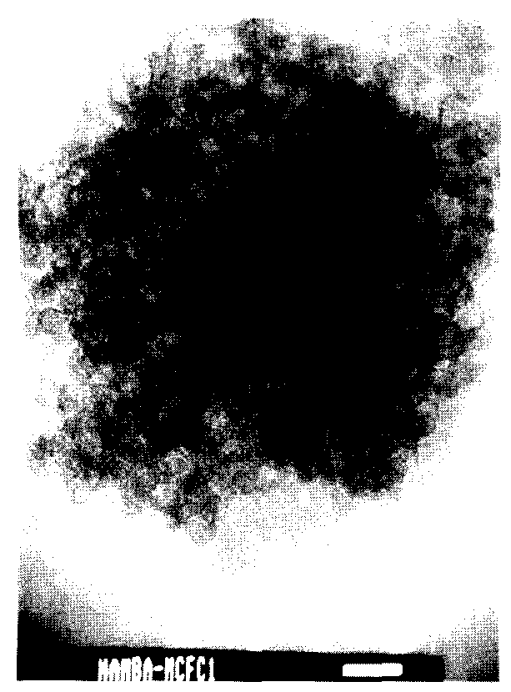

Fig. 4 Transmission electron micrograph of MCF-C. The length of white bar is $50 \mathrm{~nm}$. 
of 8.9 and $7.0 \mathrm{~nm}$ for the adsorption and desorption branches, respectively. Although the inkbottle type pore is again implied, the pore size is much smaller than MCF-Si. The BET surface area and the total pore volume are $291 \mathrm{~m}^{2} \mathrm{~g}^{-1}$ and $0.39 \mathrm{~cm}^{3} \mathrm{~g}^{-1}$, respectively.

A TEM image of mesoporous carbon is shown in Fig. 4. The aggregated spherical particles whose diameter is relatively uniform are observed. The average diameter is $30 \mathrm{~nm}$. Assuming that the density of carbon is $2 \mathrm{~g} \mathrm{~cm}^{-3}$, the calculated surface area of an average sized carbon sphere is $100 \mathrm{~m}^{2} \mathrm{~g}^{-1}$. This differs considerably from the measured BET surface area.

When mesoporous silica template is used to produce mesoporous carbon, ideally the topology of the template will be transferred inversely; the void in silica changes into the solid carbon and vice versa. However, the degree of filling-up depends on degree of dehydration, the interaction of the carbon precursor with silica surface and partially polymerised precursor in the pores. It has been reported that the impregnation of SBA-15 with the aqueous solution of furfury alcohol results in a hexagonally packed tubular carbon ${ }^{11)}$ while that of sucrose produces a hexagonally packed carbon rods. "

Assuming the hollow spheres and using the measured BET area, the diameter of the void is estimated at $24 \mathrm{~nm}$, $i . e$. the thickness of the crust is $3 \mathrm{~nm}$. The nitrogen molecules are generally not accessible to the inside of carbon particles. The mesoporous carbon obtained by the method described above consists of the aggregated spherical hollow particles. This structure resembles a foam more than MCF-Si reported by P. Schmidt-Winkel et. $a l .{ }^{14,15)}$ for which we reasonably name this carbon as mesocellular foam carbon (MCF-C).

When the concentration of sucrose was increased, $S_{B E T}$ of resultant carbon decreased without changing the pore size distributions. This result can be explained by the thickening of the wall of hollow spheres.

By using MCF-C, the MEA with a well defined structure will be prepared for a further electrochemical research to miniaturize fuel cells.

\section{Acknowledgement}

This work is financially supported by Grand-in-Aid for Scientific Research (Priority Areas Research, No. 13134203) by Ministry of Education, Culture, Sports, Science and Technology.

\section{References}

1) J. Y. Ying, C. P. Mehnert, and M. S. Wong, Angew. Chem., Int. Ed., 38, 56 (1999).

2) A. Corma, Chemi. Rev., 97, 2373 (1997).

3) J. M. Thomas, Angew. Chem., Int. Ed., 38, 3588 (1999).

4) K. Moller and T. Bein, Chem. Mater., 10, 2950 (1998).

5) J. Lee, S. Yoon, T. Hyeon, S. M. Oh, and K. B. Kim, Chem. Commun., 1999, 2177.

6) R. Ryoo, S. H. Joo, and S. Jun, J. Phys. Chem. B, 103, 7743 (1999).

7) S. Jun, S. H. Joo, R. Ryoo, M. Kruk, M. Jaroniec, Z. Liu, T. Ohsuna, and O. Terasaki, J. Am. Chem. Soc., 122, $10712(2000)$.

8) S. B. Yoon, J. Y. Kim, and J. S. Yu, Chem. Commun., 2001, 559.

9) H. J. Shin, R. Ryoo, M. Kruk, and M. Jaroniec, Chem. Commun., 2001, 349.

10) S. H. Joo, S. Jun, and R. Ryoo, Microporous Mesoporous Mater. 44-45, 153 (2001).

11) S. H. Joo, S. J. Choi, I. Oh, J. Kwak, Z. Liu, O. Terasaki, and R. Ryoo, Nature, 412, 169 (2001).

12) M. Kaneda, T. Tsubakiyama, A. Carlsson, Y. Sakamoto, T. Ohsuna, O. Terasaki, S. H. Joo, and R. Ryoo, J. Phys. Chem. B, 106, 1256 (2002).

13) J. S. Lee, S. H. Joo, and R. Ryoo, J. Am. Chem. Soc., 124, 1156 (2002).

14) P. Schmidt-Winkel, W. W. Lukens, Jr., D. Zhao, P. Yang, B. F. Chmelka, and G. D. Stucky, J. Am. Chem. Soc., 121, 254 (1999).

15) P. Schmidt-Winkel, W. W. Lukens, Jr., P. Yang, D. I. Margolese, J. S. Lettow, J. Y. Ying, and G. D. Stucky, Chem. Mater., 12, 686 (2000).

16) J. S. Lettow, Y. J. Han, P. Schmidt-Winkel, P. Yang, D. Zhao, G. D. Stucky, and J. Y. Ying, Langmuir, 16, 8291 (2000). 